\title{
Ejaculation frequency and risk of prostate cancer a systematic review
}

\begin{abstract}
Aim: The aim is to summarize the association between ejaculation frequency and risk of prostate cancer.

Methods: The Pub Med, EMBASE and Web of Science were searched to identify articles about ejaculation frequency and risk of prostate cancer without date or language restrictions (last search up to April,2016). We included prospective cohort studies about ejaculation frequency and risk of prostate cancer. We only did a descriptive analysis because of without suitable data.
\end{abstract}

Results: The result of descriptive analysis indicated that higher ejaculation frequency is associated with reducing risk of prostate cancer and lower ejaculation frequency is not associated with reducing risk of prostate cancer.

Conclusion: This systematic review showed that higher ejaculation frequency shows beneficial effect in decreasing risk of prostate cancer and lower ejaculation frequency shows no effect in decreasing risk of prostate cancer. Studies with high methodological quality are required to confirm these findings.

Keywords: ejaculation, prostate cancer, systematic review, prospective cohort studies
Volume 3 Issue 5 - 2017

\author{
Lian Liu, Fan Li, Siping Yu, Qiu Xia Liu, Xiao \\ Ling Yang, Hong Yan Sun \\ Nursing School, Southwest Medical University, China
}

Correspondence: Hong Yan Sun, Nursing School, Southwest Medical University, Luzhou, Si chuan, No. 3, 319 section of Zhong Shan Road, Jiangyang District, Luzhou 646000,China, Email sunhongyan234@I63.com

Received:September 09, 2017 | Published: September 21, 2017

\section{Introduction}

Prostate cancer (PCa), a highly ubiquitous malignancy in the whole world, includes the following risk factors advanced age, African ancestry, and family history of PCa. According to the migration studies, both environmental and lifestyle factors may be included..$^{1-3} \mathrm{~A}$ meta-analysis indicated that a more active sex life is connected with PCa. ${ }^{4}$ The following sexual activity: multiple interrelated dimensions, such as number of sexual partners, sexual orientation, gender of sexual partners, ejaculation frequency, and age at first intercourse, might become measures of different mechanisms which are possibly associated with PCa. An alternative hypothesis indicates an etiologic risk factor of $\mathrm{PCa}$ is caused by a reduced ejaculatory output in otherwise normal men, which was on the basis of the theory that infrequent ejaculation enhances the risk of $\mathrm{PCa}$ on account of retained carcinogenic secretions in the prostatic acini. According to a further hypothesis, repression of sexuality is a risk factor for $\mathrm{PCa}$ which grows out of reports of greater sexual drive coupled with deprived sexual activity. ${ }^{5,6}$ The epidemiological data about sexual activity and $\mathrm{PCa}$ are nearly only limited to case-control studies, ${ }^{4,7,8}$ which might result in methodological bias because of data about prediagnosis sexual activity collected after the diagnosis of PCa. Two studies ${ }^{9,10}$ showed that there was no association between these factors and $\mathrm{PCa}$ when age at first marriage, marital status, and number of children were as measures of sexual activity. There are no sufficient trials which can clearly address the relationship between sexual activity and risk of $\mathrm{PCa}$. The association between ejaculation frequency and risk of $\mathrm{PCa}$ is not clear. Thus, we performed a systematic review to summarize the association between ejaculation frequency and risk of PCa.

\section{Methods}

\section{Search strategy}

We searched Pub Med, EMBASE and Web of Science for studies of ejaculation frequency and risk of prostate cancer without date or language restrictions(last search up to April, 2016).Search terms were as follows: "prostate neoplasm's", "neoplasm's, prostate", "neoplasm, prostate", "prostate neoplasm", "neoplasm's, prostatic", "neoplasm, prostatic", "prostatic neoplasm", "prostate cancer", "cancer, prostate", "cancers, prostate", "prostate cancers","cancer of the prostate", "prostatic cancer", "cancer, prostatic", "cancers, prostatic", "prostatic cancers", "cancer of prostate", "ejaculations", "ejaculation". In addition, we checked reference lists of all included studies and reference lists of relevant reviews as additional search. All studies identified by this process were independently screened by two authors.

\section{Selection criteria}

Two authors independently assess the studies and all disagreements were solved by discussion. The inclusion criteria were as follows:

1. Prospective cohort study;

2. Trials about the association between ejaculation frequency and risk of $\mathrm{PCa}$,

3. Incidence of $\mathrm{PCa}$ [relative risk (RR), odds ratio or hazard rate (HR) with $95 \%$ confidence interval (CI) as one outcome.

We also excluded case reports, animal studies, letters to editors, reviews, comments, studies without original data.

\section{Data extraction}

We independently extracted the following data from eligible studies: the first author, publication year, characteristics of participant, sample size, incidence of prostate cancer [relative risk (RR), odds ratio (OR) or hazard rate (HR) with $95 \%$ confidence interval (CI)], follow up time. These data were transferred to Microsoft Excel 2010. We conscientious check these data and discrepancies were solved by our detailed discussion. 


\section{Quality assessment}

The methodological quality of cohort studies was assessed by the Newcastle-Ottawa quality assessment scale, including the following domains: the selection of participants, comparability of study groups, and outcome of interest. ${ }^{11}$ The check list includes nine items for cohort studies with every item accounting for 1 starts. High-quality studies were more than 6 stars.

\section{Statistical analysis}

We did not do a meta-analysis because of without suitable data. In this systematic review, we only did a descriptive analysis. The overall trend in the results and the degree of certainty in the results were recognized when the relevant studies in this systematic review were implicated in our focused questions.

\section{Results}

The first search identified 610 articles, of which 15 potentially relevant articles were required to evaluate full-text. Thirteen studies were excluded by the inclusion criteria and exclusion criteria. Finally, this systematic review only included 2 studies. ${ }^{12,13}$ The studies screened of this process was shown in Figure 1. Characteristics of patient were summarized in Table 1. Quality assessment of included studies were summarized in Table 2. All studies had poor methodological quality. Michael et al., ${ }^{11}$ showed that there were 1449 new cases of total PCa, including 953 organ confined cases, and 147 advanced cases of PCa, during 222426 person-years of follow-up. Most categories of ejaculation frequency were not associated with risk of PCa. High ejaculation frequency (21 or more ejaculations per month) was associated with decreasing risk of total PCa [multivariate RR, 95\%CI, $0.89,(0.73,1.10)$, ages 20 to 29 years; multivariate RR, $95 \% \mathrm{CI}, 0.68,(0.53,0.86)$, ages 40 to 49 years; multivariate $\mathrm{RR}$, $95 \% \mathrm{CI}, 0.49(0.27,0.88)$, previous year; multivariate RR, 95\% $\mathrm{CI}, 0.67$ $(0.51,0.89)$, averaged across a lifetime] compared with men reporting 4 to 7 ejaculations per month. ${ }^{12}$ Rider et al., ${ }^{13}$ showed that there were 3839 men diagnosed with PCa during 480831 person-years. PCa was less frequently diagnosed among men who had the higher ejaculation frequency (21 or more ejaculations per month, multivariable HR, $95 \% \mathrm{CI}, 0.81,(0.72,0.92), \mathrm{P}<0.0001$ for trend, ages 20 to 29 years; multivariable HR, $95 \% \mathrm{CI}, 0.78,(0.69,0.89), \mathrm{P}<0.0001$ for trend, ages 40 to 49 years] compared with men reporting 4 to 7 ejaculations per month..$^{13}$ The two trials ${ }^{12,13}$ indicated that higher ejaculation frequency is associated with reducing risk of PCa. However, lower ejaculation frequency is not associated with reducing risk of $\mathrm{PCa}$.

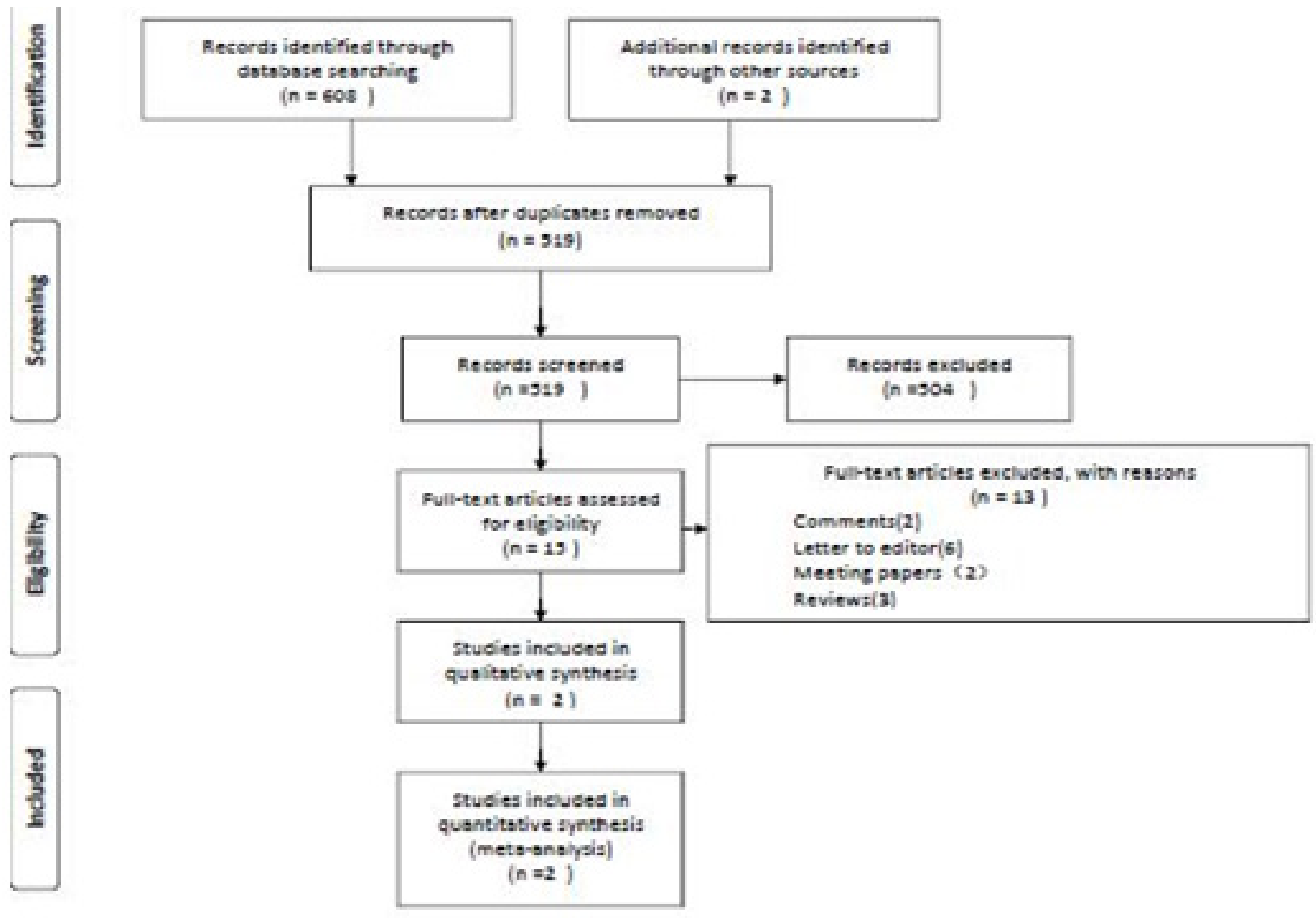

Figure I Flow diagram of included studies.

Table I Characteristics of included studies

\begin{tabular}{lllll}
\hline Study(Year) & Sample size & Age & The primary endpoint of interest & Follow up \\
\hline Michael et al. ${ }^{12}$ & 29342 & 46 to 8 lyears & Incidence of total prostate cancer & 9 years \\
${\text { Rider et al. }{ }^{13}}$ & 5 I 529 & 20 to $75 y$ years & Incidence of total prostate cancer & 19years \\
\hline
\end{tabular}


Table 2 Quality assessment of included studies on the basis of NewcastleOttawa scale

\begin{tabular}{llll}
\hline Study(Year) & Selection & Comparability & Exposure \\
\hline Michael et al. ${ }^{12}$ & $\downarrow \downarrow$ & - & $\downarrow \downarrow$ \\
Rider et al. ${ }^{13}$ & $\downarrow \downarrow$ & - & $\downarrow \downarrow$ \\
\hline
\end{tabular}

\section{Discussion}

This is the first systematic review to summarize the association between ejaculation frequency and risk of PCa. We found that higher ejaculation frequency is good for keeping risk of PCa down. Lower ejaculation frequency shows no beneficial effect in reducing risk of PCa. A meta-analysis indicated that risk of $\mathrm{PCa}$ is associated with increasing frequency of sexual activity. The mechanism about frequency of sexual activity possibly related to prostate cancer is not clear. ${ }^{4}$ Gottlieb indicated that frequent ejaculation may be related to decrease PCa. ${ }^{14}$ However, Gottlieb only summarized the results of Michael et al. ${ }^{12}$ Our findings can improve the degree of certainty in which higher ejaculation frequency is good for keeping risk of $\mathrm{PCa}$ down and low ejaculation frequency shows no beneficial effect in reducing risk of $\mathrm{PCa}$, because the findings were based on the systematic review. This systematic review have several limitations. Firstly, the included studies ${ }^{12,13}$ have poor methodological quality, which degrades reliability of systematic review. In addition, this systematic review only included two studies, and it may be lack of stable statistical significance in such a small number of studies (Figure 1). Due to lack of gray studies (presentations, unpublished data, government reports, and other traditional or nontraditional sources of evidence), there is another limitation of this systematic review. There are many unpublished studies, which may cause a potential limitation. If this systematic review gain adequate unpublished studies and gray studies, it will enhance the reliability significantly. In conclusion, the limited evidence indicates that higher ejaculation frequency shows beneficial effect in reducing risk of $\mathrm{PCa}$ and lower ejaculation frequency shows no effect in reducing risk of PCa. Man with higher ejaculation frequency may be difficult to develop PCa. Studies with high methodological quality are required to confirm this conclusion.

\section{Conflict of interest}

The author declares no conflict of interest.

\section{References}

1. Torre L, Bray F, Siegel R, et al. Global cancer statistics, 2012. CA Cancer J Clin. 2015;65(2):87-108.

2. Haenszel W, Kurihara M. Studies of Japanese migrants. I. Mortality from cancer and other diseases among Japanese in the United States. $J$ Natl Cancer Inst. 1968;40(1):43-68.

3. Pienta K, Esper P. Risk Factors for Prostate Cancer. Ann Intern Med. 1993;118(10):793-803.

4. Dennis LK, Dawson DV. Meta-Analysis of Measures of Sexual Activity and Prostate Cancer. Epidemiology. 2002;13(1):72-79.

5. Isaacs J. Prostatic structure and function in relation to the etiology of prostatic cancer. The Prostate. 1983;4(4):351-366.

6. Rotkin ID. Studies in the epidemiology of prostatic cancer: expanded sampling. Cancer Treat Rep. 1977;61(2):173-180.

7. Strickler HD, Goedert JJ. Sexual behavior and evidence for an infectious cause of prostate cancer. Epidemiol Rev. 2001;23(1):144-151.

8. Oliver RT. Sexual factors and prostate cancer. BJU Int. 2004;93(3):422.

9. Mills P, Beeson W, Phillips R, et al. Cohort study of diet, lifestyle, and prostate cancer in adventist men. Cancer. 1989;64(3):598-604.

10. Severson R, Nomura A, Grove J, et al. A Prospective Study of Demographics, Diet, and Prostate Cancer among Men of Japanese Ancestry in Hawaii. Cancer Res. 1989;49(7):1857-1860.

11. Hartling L, Milne A, Hamm MP, et al. Testing the Newcastle Ottawa Scale showed low reliability between individual reviewers. $J$ Clin Epidemiol. 2013;66(9):982-993.

12. Leitzmann MF, Platz EA, Stampfer MJ, et al. Ejaculation frequency and subsequent risk of prostate cancer. JAMA. 2004;291(13):1578-1586.

13. Rider JR, Wilson KM, Sinnott JA, et al. Ejaculation Frequency and Risk of Prostate Cancer: Updated Results with an Additional Decade of Follow-up. Eur Urol. 2016;70(6):974-982.

14. Gottlieb S. Frequent ejaculation may be linked to decreased risk of prostate cancer. British Medical Journal. 2004;328(7444):851-851.

\section{Acknowledgements}

None. 